Sami Hiltunen, Arttu Mankinen, Muhammad Asadullah Javed, Susanna Ahola, Martti Venäläinen and Ville-Veikko Telkki*

\title{
Characterization of the decay process of Scots pine caused by Coniophora puteana using NMR and MRI
}

https://doi.org/10.1515/hf-2019-0246

Received October 4, 2019; accepted February 11, 2020

\begin{abstract}
Wood decay is an economically significant process, as it is one of the major causes of wood deterioration in buildings. In this study, the decay process of Scots pine (Pinus sylvestris) samples caused by cellar fungus (Coniophora puteana) was followed by nuclear magnetic resonance (NMR) spectroscopy and magnetic resonance imaging (MRI) methods. Altogether, 30 wood sample pieces were exposed to fungus for 10 weeks. Based on the decrease of the dry mass, the samples were categorized into three classes: decomposed (mass decrease $50-70 \%$ ), slightly decomposed (10-50\%), and nondecomposed $(<10 \%)$. MRI made it possible to identify the active regions of fungus inside the wood samples based on the signal of free water brought by the fungus and arisen from the decomposition of wood carbohydrates. MRI implies that free water is not only created by the decay process, but fungal hyphae also transports a significant amount of water into the sample. Two-dimensional ${ }^{1} \mathrm{H} T_{1} T_{2}$ relaxation correlation NMR measurements provided detailed information about the changes in the microstructure of wood due to fungal decomposition. Overall, this study paves the way for noninvasive NMR and MRI detection of fungal decay at early stages as well as the related structural changes.
\end{abstract}

Keywords: brown rot, MRI, NMR spectroscopy, pine wood, relaxation

\footnotetext{
*Corresponding author: Ville-Veikko Telkki, NMR Research Unit, Faculty of Science, University of Oulu, P.O. Box 3000, FI-90014 Oulu, Finland, e-mail: ville-veikko.telkki@oulu.fi

Sami Hiltunen, Arttu Mankinen, Muhammad Asadullah Javed and Susanna Ahola: NMR Research Unit, Faculty of Science, University of Oulu, P.O. Box 3000, Fl-90014 Oulu, Finland

Martti Venäläinen: Natural Resources Institute Finland (Luke), Vipusenkuja 5, Fl-57200 Savonlinna, Finland
}

\section{Introduction}

Wood is a hygroscopic material that adsorbs moisture in humid conditions (Simpson 2007). Water adsorbed in cell walls is called bound water, whereas water in larger cavities, such as lumens of tracheid cells, is called free water (Kettunen 2006). In dry wood, moisture is first adsorbed into cell walls. As the bound water content increases, wood swells, its mechanical strength decreases, thermal and electrical conductivities increase, and the rate of bound water diffusion increases. Free water appears in the wood only close to the cell wall saturation point, which is called the fiber saturation point (FSP). Beyond this point, the amount of free water increases, but the physical properties of wood change only slightly with increasing moisture content (MC; Siau 1984; Skaar 1988). For pine wood, the MC corresponding to the FSP is $25 \%$ to $30 \%$.

Fungal decay is one of the most significant causes of wood deterioration in buildings (Tura et al. 2016). To enable the fungal metabolic activity, the MC of wood has to be high enough, i.e. close to or above FSP (Griffin 1977; Zabel and Morrell 1992). The efficiency of the decay process depends on many environmental factors, such as temperature and availability of nutrients and oxygen (Zabel and Morrell 1992; Ringman et al. 2019).

Cellar fungus Coniophora puteana is a very common threat for softwood in buildings. It is a brown-rot fungus that decomposes cellulose and hemicellulose of wood and leaves only the modified lignin as residue. It can cause a mass loss of wood up to 70\% (Hyde and Wood 1997; Irbe et al. 2006; Novaes et al. 2010). The optimal MC for cellar fungus is $50 \%$ to $60 \%$, but it is effective in a range of $15 \%$ to $80 \%$. The fungus is active at the temperature range of $0^{\circ} \mathrm{C}$ to $45^{\circ} \mathrm{C}$ (Williams and Hale 2003; Tura et al. 2016).

Nuclear magnetic resonance (NMR) spectroscopy is one of the most powerful tools in chemical analysis (Keeler 2010). The NMR frequency of nuclear spins depends on the structure of the electron cloud surrounding the nucleus as well as other nearby spins. Therefore, spins in different functional groups of a molecule resonate 
at different frequencies and the NMR spectrum is like a fingerprint of the molecule. The $T_{1}$ and $T_{2}$ relaxation times of the spins reflect the mobility of the molecules (especially rotational motion). The most well-known application of NMR, magnetic resonance imaging (MRI), enables one to determine the spatial distribution of spins not only in medical context but also in materials research (Brown et al. 2016).

NMR has been broadly exploited also in wood research. The ${ }^{1} \mathrm{H}$ NMR signal of solid wood is very broad, of the order of $100 \mathrm{ppm}$, and its $T_{2}$ relaxation time is very short (Dvinskikh et al. 2011a; Terenzi et al. 2013). Therefore, it is readily distinguished from the signal of moisture, which is characterized by much narrower line and longer $T_{2}{ }^{1} \mathrm{H}$ NMR can be used to quantify the MC of wood (Sharp et al. 1978; Quick et al. 1990; Hartley et al. 1994; Labbé et al. 2002; Merela et al. 2009). ${ }^{1} \mathrm{H}$ relaxation measurements provide means to resolve and quantify the free and bound water components in wood, as the $T_{2}$ of bound water is much shorter $(\sim 1 \mathrm{~ms})$ than that of free water (10-100 ms; Riggin et al. 1979; Menon et al. 1987, 1989; Fantazzini et al. 2006; Almeida et al. 2007; Telkki et al. 2013; Zhang et al. 2013; Kekkonen et al. 2014, Beck et al. 2017; Zhou et al. 2018). $T_{2}$ also reflects the size of the cavities; the shorter $T_{2}$ is, the smaller is the cavity (Menon et al. 1989; Javed et al. 2015). The size distribution of micropores in the cell walls as well as lumens has been investigated by NMR diffusion and cryoporometry methods (Kekkonen et al. 2009, 2014). Solidstate NMR techniques reveal the details of the chemical composition of wood (Sivonen et al. 2002; Koenig et al. 2010). Time-domain NMR has also been exploited in the investigation of drying and water absorption of (modified) wood (Hoffmeyer et al. 2011; Thygesen and Elder 2009) as well as in the investigation of natural and artificial aging of wood (Rostom et al. 2019). Twodimensional (2D) $T_{1}-T_{2}$ correlation experiments significantly improve the resolution and information content of the analysis; for example, they enable the resolution of two types of bound water in cell walls (Cox et al. 2010; Bonnet et al. 2017; Rostom et al. 2019).

${ }^{1} \mathrm{H}$ MRI reflects predominantly the spatial distribution of free water in wood, as the signal of bound water and wood macromolecules is mostly decayed before detection (Quick et al. 1990; Pearce et al. 1997; Rosenkilde and Glover 2002; van Houts et al. 2004, 2006; Hameury and Sterley 2006; MacMillan et al. 2002; Wang and Chang 2007; Hernández and Cáceres 2010; Mazela et al. 2014; Javed et al. 2015; Ibach et al. 2016; Zupanc et al. 2019). It is also possible to measure relaxation-weighted images or $T_{2}$ relaxation maps to achieve better contrast in between free water in different wood structures (Araujo et al. 1992; Telkki et al. 2013; Kekkonen et al. 2014; Javed et al. 2015).

NMR has also been used to determine the structural changes in wood caused by the decay process. Gilardi et al. (1994) observed increased $T_{1}$ and $T_{2}$ relaxation times in wood due to higher degree of mobility in degraded wood. Onuchin et al. (2018) exploited $T_{2}^{\star}$ contrast to determine the relative amounts of decayed and sound wood. Beck et al. (2018) studied wood-water relations before and after brown-rot decay using lowfield nuclear magnetic resonance relaxometry on watersaturated samples. The amounts of lignin and cellulose in degraded wood have been quantified by ${ }^{13} \mathrm{C}$ crosspolarized magic angle spinning technique (Gilardi et al. 1995). Müller et al. (2001) showed that the fungal decay of wood can be detected by MRI. The authors hypothesized that the method is based on the fact that brownrot fungi decompose cell wall carbohydrates into $\mathrm{CO}_{2}$ and water. Consequently, the $\mathrm{MC}$ content in the active regions of fungus increases above the FSP, and the produced free water becomes visible in MRI, whereas other regions remain invisible. Initially, primarily low molecular weight sugars within the cell lumens are respired, with no strength, mass, or density changes. However, the authors concluded that MRI enables the detection of the initial stage of the decomposition.

In this study, the decay process of 30 wood samples exposed to cellar fungus was monitored for 10 weeks. Based on the overall decrease of the dry mass of the samples, they were categorized into three classes: decomposed, slightly decomposed, and nondecomposed. With MRI, the progress of fungal activity inside the samples was directly observed based on the assumption that the occurrence of free water inside the samples can be explained only by water transportation from outside by fungal hyphae or fungal degradation of carbohydrates. $T_{1}-T_{2}$ correlation measurements provided detailed, complementary information about the changes in the microstructure of wood.

\section{Materials and methods}

Materials were provided by the Natural Resources Institute Finland (Luke), and they were derived from Punkaharju $\left(61^{\circ} 81^{\prime} \mathrm{N}, 2^{\circ} 32^{\prime} \mathrm{E}\right)$, Finland. There were 30 nonextracted autoclave-sterilized Scots pine (Pinus sylvestris) sapwood pieces, which were serially numbered from 1 to 30 . The pieces had rectangular cuboid shape with dimensions of about $6 \times 6 \times 30 \mathrm{~mm}$. The longest dimension of the samples was along the longitudinal direction of wood. Aggressively growing 
cellar fungus (Coniophora puteana; strain BAM 112) inoculated in Petri dishes with malt extract agar as media (European standard EN113 1996) was used for decomposing the wood pieces. There was a sterile supporting net structure in the dishes for preventing the samples to absorb water directly from the wet agar.

The samples were dried in an oven at $60^{\circ} \mathrm{C}$ for $48 \mathrm{~h}$, and the dry masses (at $\sim 2 \% \mathrm{MC}$ ) were weighed before the start of the decay process. Then, the samples were inserted in the dishes, three samples in one dish, in numerical order. Dishes were kept in an incubation cabinet at a temperature of $24^{\circ} \mathrm{C}$ and relative humidity of $70 \%$. Figure 1 shows a representative example of a dish in the beginning of the decay process (left) and after 1 week incubation (right). After 1 week, all the samples were covered by the fungus.

${ }^{1} \mathrm{H}$ NMR and MRI as well as gravimetric measurements were performed for two or three dishes for 1, 2, 4, 6, 8, and 10 weeks after the beginning of the decay process. Some samples were measured many times to follow the decay process thoroughly, whereas the other samples were measured only once to avoid interruptions in the decay process. Only one dish was out of the cabinet at the same time to minimize interruptions and contamination. The treated dish was taken to a laminar flow cabinet, where it was opened, and the hypha was wiped off from the samples. After weighing a sample, it was inserted in a $10 \mathrm{~mm}$ diameter NMR tube. Teflon pieces on the top and bottom fixed the sample inside the NMR tube. The NMR tube was sealed with parafilm for measurements. After the NMR and MRI measurements, the pieces were placed back in dishes in the same position as before the measurements and taken back to the incubation cabinet.

During the decay process, some of the dishes began to dry despite the high humidity in the incubation cabinet. To stop the drying, those dishes were almost fully sealed with parafilm to prevent the moisture evaporation. Only a little aeration hole was left open. Samples 1 to 3, 7 to 18 , and 25 to 27 were treated this way. However, it turned out to be very useful to have samples with different outcomes in their decay processes; it enabled us to identify the NMR and MRI signatures of each classes (decomposed, slightly decomposed, and nondecomposed). After the last NMR and MRI measurements, the samples were dried in an oven at $60^{\circ} \mathrm{C}$ for $48 \mathrm{~h}$ and their final dry weight was measured.

The NMR and MRI measurements were carried out by Bruker Avance III 300 spectrometer equipped with a microimaging unit with $\mathrm{x}$-, $\mathrm{y}$-, and z-gradients. A $10 \mathrm{~mm}{ }^{1} \mathrm{H}$ RF insert as well as TopSpin and Paravision software (Bruker) were used in the measurements.

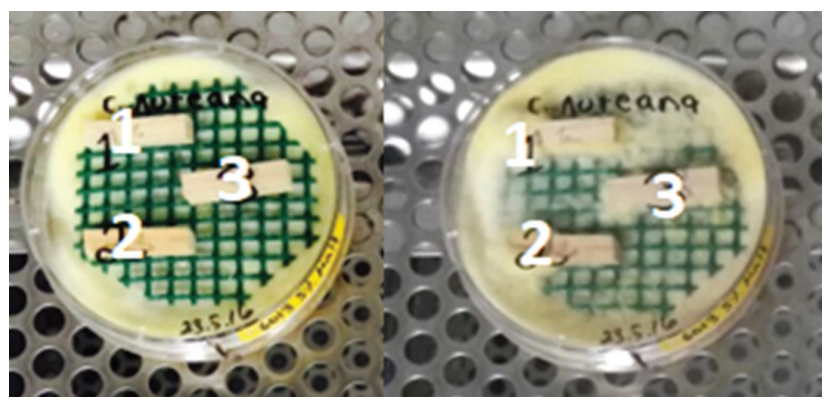

Figure 1: An example of a dish including the wood samples at the beginning of the decay process (left) and after 1 week incubation (right).

In 1 week, hyphae have grown around the samples.
First, the position of the sample was checked and adjusted by measuring three orthogonal slices by a gradient-echo imaging method. Thereafter, an axial slice (parallel to the radial-tangential plane) was measured using a multiecho spin echo imaging method with the following parameters: number of echoes, 8; echo time, $12.4 \mathrm{~ms}$; field-of-view, $1 \mathrm{~cm}$; spatial resolution, $125 \mu \mathrm{m}(80 \times 80$ matrix $)$; slice thickness, $2 \mathrm{~mm}$; repetition time, $1.8 \mathrm{~s}$; number of averages, 2; and scan time, $4 \mathrm{~min} 48 \mathrm{~s}$. The slices corresponding to the first echo are shown as spin-density images in Results and discussion, whereas all the eight echoes were used to reconstruct $T_{2}$ maps.

In addition to MRI experiments, spectroscopic $2 \mathrm{D} T_{1}-T_{2}$ relaxation correlation maps were measured by the inversion recovery (IR)Carr-Purcell-Meiboom-Gill (CPMG) sequence (Peemoeller et al. 1981; Song et al. 2002). The sequence includes an IR part (Vold et al. 1968) followed by the CPMG loop (Carr and Purcell 1954; Meiboom and Gill 1958). The number of echoes in the CPMG loop was 512 and the echo time was $0.7 \mathrm{~ms}$. IR-delay varied from 0.001 to $5 \mathrm{~s}$ (increasing logarithmically), and the sequence was repeated with 24 delays. The relaxation delay was at least three times the longest $T_{1}$ in the sample (individually acquired). Pulse lengths were $17.25 \mu$ s for $90^{\circ}$ and $34.5 \mu$ s for $180^{\circ}$ pulses. The number of scans was 4 , and the experiment time was about $4 \mathrm{~min}$.

The measurement data were processed by MATLAB. The MATLAB-based Laplace inversion program was provided by the late Prof. P. Callaghan. Masses of the samples were measured before and after all the NMR and MRI experiments, and the mass decrease during the experiments was insignificant. This confirms that the drying of samples during the experiments was minor.

\section{Results and discussion}

\section{Decomposition level based on gravimetry}

The relative mass changes of the samples during the decay process are shown in Table 1 . The percentage of the mass change was calculated by the following equation:

$$
\Delta m=\frac{m_{f}-m_{i}}{m_{i}} \times 100 \%
$$

where $m_{i}$ and $m_{f}$ are the initial and final dry masses of the wood samples (in Figure 2, $m_{f}$ is the wet mass of the wood sample before MRI scan). The observed mass change varied from $+8 \%$ to $-70 \%$. There were several samples whose mass changed almost $-70 \%$. The mass change for some other samples was negligible, indicating that the decay process did not progress for too dry conditions or some other reasons. There were also some samples with the mass change between these extremes, showing a moderate effect of fungus.

Based on the gravimetric data, the wood samples were divided into three classes (see Table 1): decomposed ( $\Delta m$ between $-70 \%$ and $-50 \%$ ), slightly decomposed ( $\Delta m$ between $-50 \%$ and $-20 \%$ ), and nondecomposed 
Table 1: Classification of the samples by mass change $\Delta m$ [percentage difference between the final and initial dry mass of the wood samples; see Equation (1)].

\begin{tabular}{|c|c|c|c|c|}
\hline Sample \# & $\Delta m(\%)$ & Class & Initial density $\left(\mathrm{kg} \mathrm{m}^{-3}\right)$ & Final water content (\%) \\
\hline 3 & -70 & Decomposed & 500 & 157 \\
\hline 1 & -68 & Decomposed & 499 & 165 \\
\hline 14 & -64 & Decomposed & 472 & \\
\hline 29 & -64 & Decomposed & 510 & 206 \\
\hline 11 & -63 & Decomposed & 505 & \\
\hline 26 & -62 & Decomposed & 523 & 75 \\
\hline 10 & -61 & Decomposed & 486 & \\
\hline 25 & -61 & Decomposed & 537 & 99 \\
\hline 19 & -61 & Decomposed & 468 & \\
\hline 23 & -60 & Decomposed & 470 & \\
\hline 30 & -60 & Decomposed & 530 & 245 \\
\hline 13 & -60 & Decomposed & 484 & \\
\hline 2 & -58 & Decomposed & 433 & 141 \\
\hline 22 & -58 & Decomposed & 459 & \\
\hline 21 & -57 & Decomposed & 492 & \\
\hline 12 & -50 & Decomposed & 463 & \\
\hline 27 & -50 & Decomposed & 460 & 105 \\
\hline 24 & -43 & Slightly decomposed & 491 & \\
\hline 15 & -41 & Slightly decomposed & 456 & \\
\hline 28 & -33 & $\begin{array}{l}\text { Slightly decomposed } \\
\text { (saturated) }^{\mathrm{a}}\end{array}$ & 481 & 237 \\
\hline 4 & -28 & Slightly decomposed & 439 & \\
\hline 5 & -25 & Slightly decomposed & 497 & \\
\hline 20 & -20 & Slightly decomposed & 490 & \\
\hline 8 & -5 & Nondecomposed & 493 & \\
\hline 7 & -4 & Nondecomposed & 555 & \\
\hline 18 & -1 & Nondecomposed & 503 & \\
\hline 16 & 3 & Nondecomposed & 419 & \\
\hline 6 & 4 & Nondecomposed & 453 & \\
\hline 9 & 6 & Nondecomposed & 452 & \\
\hline 17 & 8 & Nondecomposed & 466 & \\
\hline
\end{tabular}

Classes: decomposed ( $\Delta m$ between $-70 \%$ and $-50 \%$ ), slightly decomposed ( $\Delta m$ between $-50 \%$ and $-20 \%$ ), and nondecomposed $(\Delta m$ between $-20 \%$ and $+8 \%$ ). The initial density of wood is calculated by dividing the initial dry mass by the volume of the sample calculated from the sample dimensions. The final water content is calculated for the samples measured and weighed on week 10 . It is calculated by dividing the mass of moisture by the final dry mass of the sample. ${ }^{a} \mathrm{MRI}$ showed that sample 28 was water saturated at the end of the decay process (on week 10 but not on week 2).

( $\Delta m$ between $-20 \%$ and $+8 \%$ ). Of the 30 samples, 17 belonged to the decomposed class. Those samples were brownish and fragile as the brown rot decomposed almost all cellulose and hemicellulose, and mostly modified lignin was left (Hyde and Wood 1997; Kettunen 2006; Novaes et al. 2010). The decomposition of the cellulose and hemicellulose was not complete in the slightly decomposed samples. The nondecomposed pieces did not lose mass almost at all or they even gained more mass during the experiment. The latter could be explained by experimental errors, incomplete drying process after the experiment, or some fungus remaining in the exterior of those samples.

\section{Visualization of the decay process by MRI}

Figure 2 shows representative ${ }^{1} \mathrm{H}$ spin-density images of the samples belonging to each decomposition class as a function of the decay time. As explained in Materials and methods, the initial MC of the samples was about $2 \%$, i.e. well below FSP; therefore, there was no free water in wood at the beginning of the decay process. At a relative humidity of $70 \%$, the samples began to absorb water. However, adsorption from air could not raise the MC above FSP. When a significant amount of free water is observed in the samples, the water has to be transported into the sample by fungal hyphae or it is created by fungal degradation 

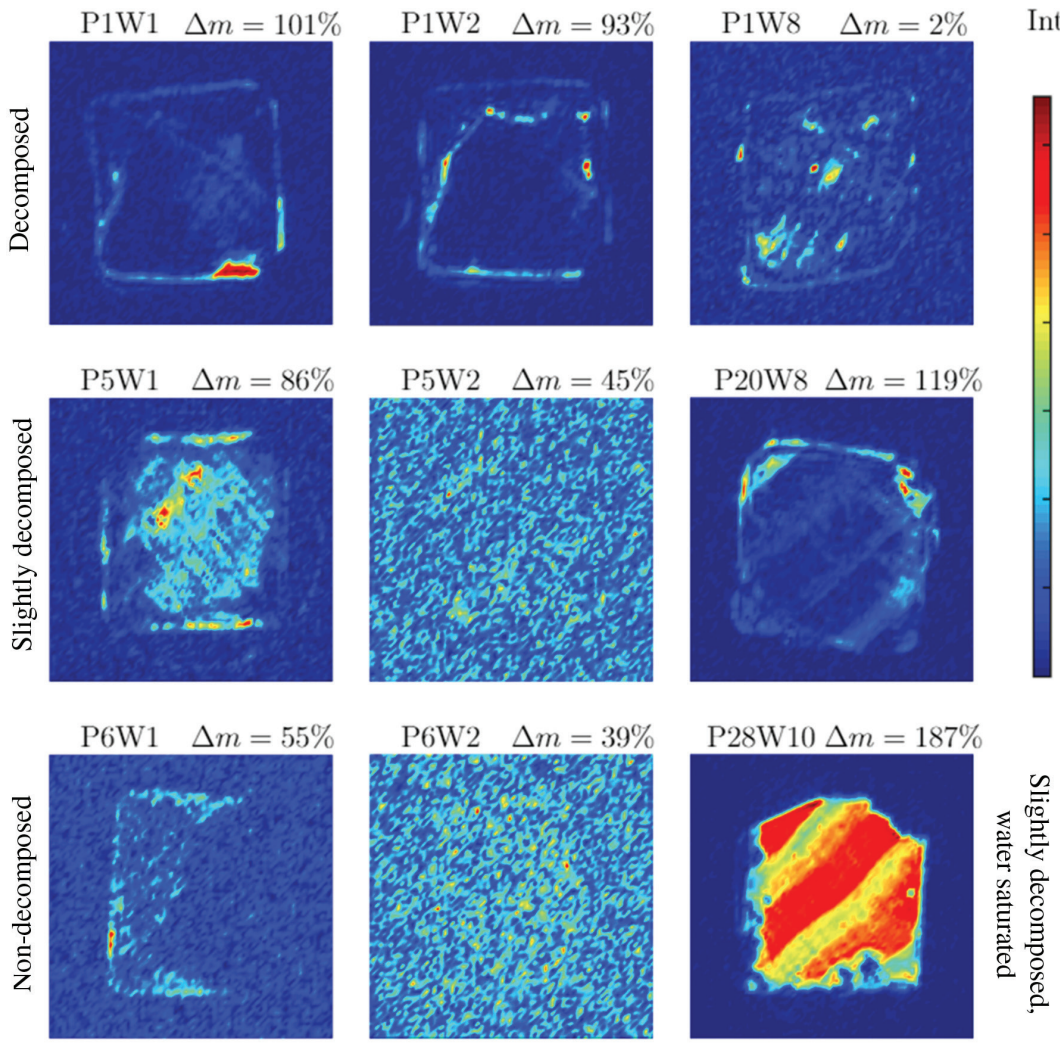

Int

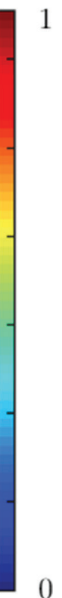

()

Figure 2: Spin-density MRI of the decomposing wood samples.

The images are $2 \mathrm{~mm}$ axial slices (radial-tangential plane) from the center of the samples. Label P1W1 refers to piece 1 on week 1 . The first row depicts decomposed samples, the second row depicts slightly decomposed samples, and the third row depicts nondecomposed samples (except for the image on the bottom, right, which depicts the slightly decomposed, water-saturated sample). The intensity is proportional to the amount of free water molecules, and the maximum intensity of each image is scaled to be 1 . Therefore, the amplitudes of different images are not comparable. The images including mostly noise indicate that the amount of free water is negligible in those samples. The regions of free water signal show the locations of active decay process. The field of view is $10 \times 10 \mathrm{~mm}$.

of carbohydrates. These processes cause a local, discontinuous, strong signal of free water, which can be readily disentangled from weak and smooth background signal of bound water or small amount of free water, which may exist even below FSP (Telkki et al. 2013).

The image of the decomposed sample (piece 1) measured during the first week (up, left) shows a strong signal of free water at the edges of the sample, indicating that initially the fungus was active in those regions, decomposing wood carbohydrates into water and $\mathrm{CO}_{2}$ (Müller et al. 2001). However, the signal is so strong that we suggest that it is not only caused by the water arising from the decomposition, as Müller et al. hypothesized, but also most probably it includes the contribution of water brought by fungus as well; a maximum of $56 \%$ of the mass of the decomposed cellulose and hemicellulose is converted to water, and in the initial stage of the decomposition process, this mechanism could not cause a significant increase of the amount of free water.
The relative mass changes $(\Delta m)$ calculated by Equation (1) are also given in Figure 2. Here, $m_{f}$ corresponds to the mass of the sample before the NMR experiments, whereas $m_{i}$ is the initial dry mass. Before the fungal decay, $\Delta m$ is equal to the MC of the sample. Fungal decay decreases the mass of solid wood and the fungus increases the total mass of wood. As the previous effect is expected to be dominant, $\Delta m$ represents the lower limit of MC, i.e. MC is larger than $\Delta m$ during the decay process.

At this point, the mass of the wood sample had increased by $101 \%$ compared to the dry mass mostly due to the increased MC of the sample but partially also due to the mass of the fungus. Based on the mass increase, it is obvious that the MC of the sample was much higher than the FSP; therefore, the gravimetric data confirmed that there was a significant amount of free water in the sample. Not all the free water could be in the regions of the most intense MRI signals at the edges of the sample; free water had to exist in other regions as well. Indeed, there is some 
free water signal visible all around the sample shown as a light blue color in the image. The $T_{2}$ relaxation time of free water covers a broad range, from 10 to $100 \mathrm{~ms}$ (see also Figure 4). As the echo time in MRI experiments was $12.4 \mathrm{~ms}$, only about $30 \%$ of the signal of the shortest $T_{2}$ component of free water (corresponding to small cavities) was detected, whereas the same weighting factor for the longest $T_{2}$ component was $90 \%$. As the relaxation time of bound water is much smaller ( $0.5-5 \mathrm{~ms}$, see below) than the echo time, its contribution to the observed signal was minor.

The image from week 2 (up, middle) reveals that the fungus had penetrated deeper inside the sample. The mass of the sample was slightly lower than on week 1 $(\Delta m=93 \%)$, but, anyway, the MC remained high. The image from week 8 shows bright free water spots in the center of the sample, showing that the decomposition process was almost completed. The signal-to-noise ratio is lower than in the previous images because of the lowered activity of the fungus (and therefore lowered amount of free water) at the end of the process. The lowered free water content and decomposition of the wood is reflected in $\Delta m$, which was dropped to $2 \%$. According to Table 1 , the overall dry mass change of piece 1 was $-68 \%$. As $\Delta m$ on week 8 was $2 \%$, the sample included a significant amount of moisture, potentially located in the water-adsorbing micropores created during the decomposition process as proposed by Karppanen et al. (2008). Most probably, the $T_{2}$ of water in the micropores is so short that the corresponding signal is significantly attenuated in MRI. However, based on the observations described above, we interpreted that the fungus activity is associated with the increase of local concentration of free water observable with MRI, and only after the activity has been stopped, free water can completely disappear.

The image of the slightly decomposed sample (piece 5 , middle, left) measured on week 1 also shows a significant amount of free water in the sample, and the gravimetric analysis confirms the high $\mathrm{MC}(\Delta m=86 \%)$. However, the image from the second week (middle, middle) does not show any free water signal but noise only. This significantly decreased mass $(\Delta m=45 \%)$ imply that the decay process was interrupted due to too dry conditions. The image of another slightly decayed sample (piece 20) measured on week 8 shows brighter free water signal only in the outer parts of the sample, indicating that most of the sample volume was intact.

The images of the nondecomposed piece 6 measured in both weeks 1 and 2 (bottom, left and middle) are almost lacking the free water signal. The weight of the sample was also low ( $\Delta m=55 \%$ and $39 \%$ ), suggesting that the decay process did not start at all due to the low MC.
One of the slightly decomposed samples (piece 28) showed extremely high free water signal (bottom, right) along with very high mass $(\Delta m=187 \%)$ on week 10 . We interpreted that this sample was almost fully saturated with water (perhaps due to the dropping of condensed water from the dish cover), and the fungal metabolism was ceased due to the lack of oxygen. The clear regions of annual rings (early and late wood) visible in the images visualizes also that the fungus did not change the structure of wood significantly. However, the observed decrease of the dry mass ( $-33 \%$; see Table 1$)$ indicates that there had been active degradation process in the beginning when the sample was not water saturated.

The bright spots in Figure 2 indicating the active regions of fungus are rather small $(\leq 1 \mathrm{~mm})$ compared to the overall size of the sample pieces as well as the distances water can diffuse to during the decay process. Therefore, the extra water may be diluted to the close surroundings due to diffusion after the activity of the fungus has finished and it does not necessarily need to be removed from the wood. The fungus may also transfer some of the extra water to the new active region.

Figure 3 shows the corresponding $T_{2}$ maps of the same samples. The maps are highlighting the distribution of free water in a similar way as the spin-density images in Figure 2. However, the contrast is slightly different. Free water in the active regions of the fungus appear to have $T_{2}$ of $\sim 20 \mathrm{~ms}$ (light blue color), which is longer than the echo time (12.4 ms) in the spin-density images. In the slightly decomposed, water-saturated sample (bottom, right), free water in early wood has significantly longer $T_{2}$ than free water in late wood due to smaller lumen sizes in the latter part. Small dots visible in the late wood parts arise from free water in longitudinal resin channels (Javed et al. 2015). It is noted that the randomly appearing reddish regions in the $T_{2}$ maps are noise (not water components with a long $T_{2}$ ).

MRI observations can be summarized in the following way: small intense regions of free water signal in the spindensity images and $T_{2}$ maps highlight the active regions of the fungus; if there is no free water visible in the images or maps, the fungus is not active; if an intense free water signal is continuously visible over the whole sample, the sample is water saturated and the fungus is not active due to the lack of oxygen delivery.

\section{Free and bound water during the decay process}

Bound water cannot be observed in MRI due to its short $T_{2}$. However, both bound and free water can be detected in 

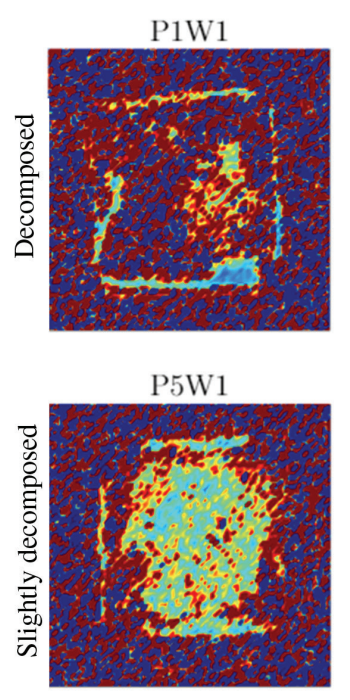

P6W1
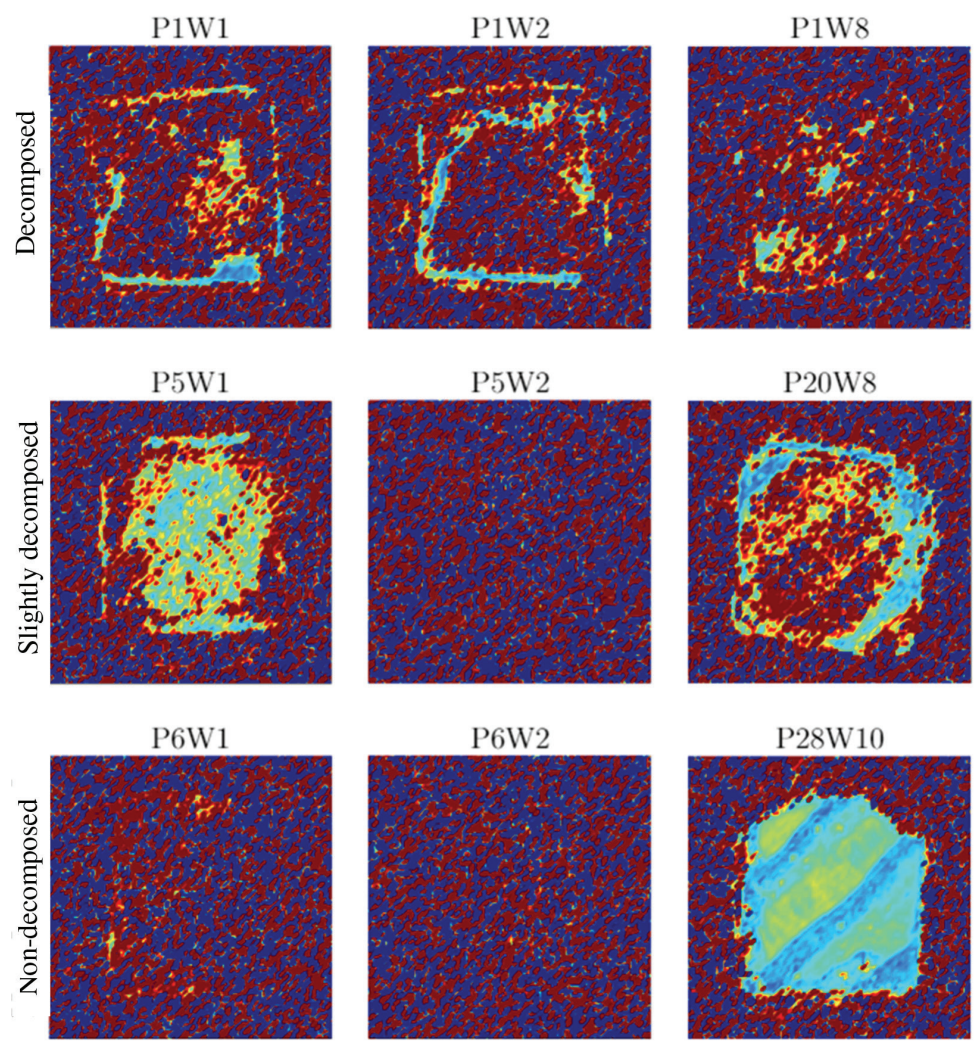

$T_{2}(\mathrm{~ms})$
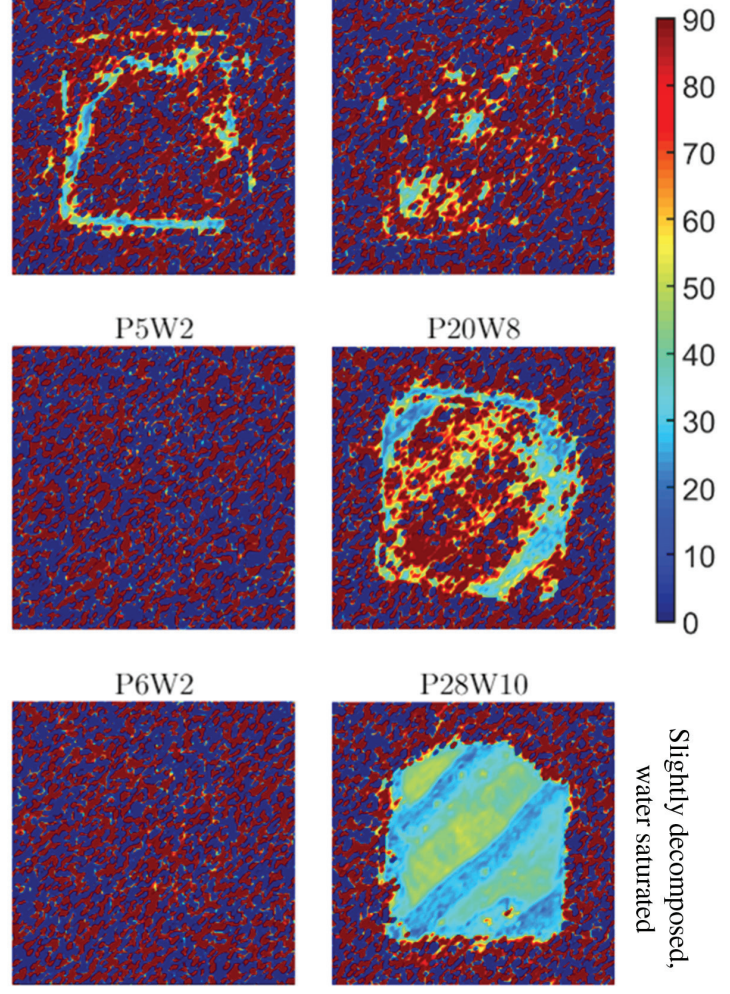

P28W10

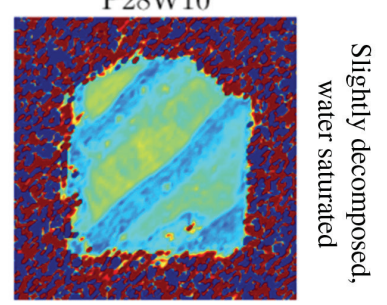

Figure 3: $T_{2}$ MRI of the same samples in Figure 2.

Note that the dark red background is noise.

the relaxometry experiments (without spatial resolution). Therefore, these experiments provide useful complementary information about the changes in the microstructure of wood due to the decay process, as it also changes the mobility of both bound and free water.
Figure 4 shows representative ${ }^{1} \mathrm{H} T_{1}-T_{2}$ correlation maps and $T_{2}$ distributions of the samples belonging to each decomposition class. The nondecomposed sample (piece 6, week 2) shows three peaks in both $T_{1}-T_{2}$ map and $T_{2}$ distribution. The most intense signal has the shortest $T_{2}$

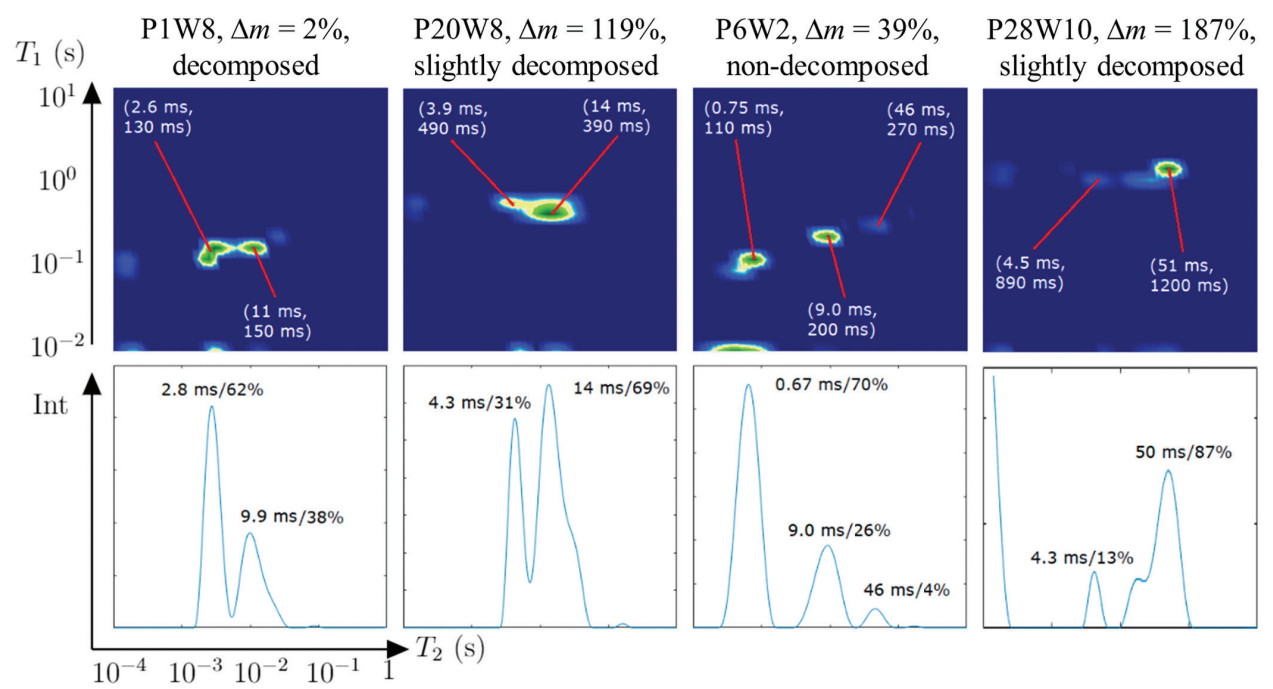

Figure 4: $T_{1}-T_{2}$ relaxation correlation maps (top) and $T_{2}$ relaxation distributions of the wood samples.

$T_{2}$ and $T_{1}$ relaxation times of the tops of the peaks are shown in the $T_{1}-T_{2}$ maps. Furthermore, $T_{2}$ values and relative integrals of the peaks are given in the $T_{2}$ distributions. 
( $\sim 0.7 \mathrm{~ms})$ and $T_{1}(110 \mathrm{~ms})$, and it arises from bound water (Telkki et al. 2013; Kekkonen et al. 2014). The second intense signal owns intermediate relaxation time values ( $T_{2}=9 \mathrm{~ms}$ and $T_{1}=200 \mathrm{~ms}$ ) and the smallest signal the longest $\left(T_{2}=46 \mathrm{~ms}\right.$ and $\left.T_{1}=270 \mathrm{~ms}\right)$. These two signals originate from free water. One possible interpretation is that the shorter $T_{2}$ component arises from free water in late wood tracheid cells (small lumens) and the longer one from early wood (large lumens), as, according to the surface relaxation model of Brownstein and Tarr (1979), $T_{2}$ is directly proportional to the pore size. Furthermore, our earlier MRI $T_{2}$ maps (and Figure 3) showed shorter $T_{2}$ values of free water in late wood than in early wood of pine (Javed et al. 2015); the values differed approximately by the factor of 2, which is equal to the lumen size difference (Kettunen 2006). In contrast, Fredriksson and Thygesen (2017) observed two free water $T_{2}$ components from separately measured early and late wood spruce pieces and interpreted that the longer $T_{2}$ component arose from tracheid lumina, whereas the shorter one from the pits and ray cell lumina. Furthermore, extractives and organic compounds may also change $T_{2}$ values (Labbé et al. 2006). Therefore, it is not possible to make conclusive interpretations of the origins of the two free water components.

The slightly decomposed wood sample (piece 20, week 8 ) shows only two peaks in narrower region in both $T_{2}$ and $T_{1}$ directions. We interpreted that this is a consequence of the partial destruction of the structure of wood. As the cell walls decomposed partially, bound water had higher mobility, and $T_{2}$ of bound water became longer $\left(T_{2} \approx 4 \mathrm{~ms}\right.$; Beck et al. 2018). Due to the same reason, the two free water pools are not well resolved anymore. $T_{1}$ values of bound and free water became almost equal ( $\sim 400 \mathrm{~ms})$. A potential explanation is that diffusion-driven exchange time between these pools became short compared to $T_{1}$ due to the decomposition, making $T_{1}$ values equal. In contrast, the exchange time was long compared to $T_{2}$, as separate peaks of bound and free water were observed in the $T_{2}$ direction. In addition, the signals of bound and free water pools may be partially overlapping with the signal of water-adsorbing micropores created during the decomposition process (Karppanen et al. 2008). $T_{1}$ value of water in the slightly decomposed wood sample is higher than that of any component in the nondecomposed sample. Most probably, this is associated with the higher relative free water content due to MC.

In the decomposed sample (piece 1 , week 8), the peaks are even narrower region in the $T_{2}$ direction than in the slightly decomposed sample due to the decomposition of cell walls, and $T_{1}$ values are equal due to the accelerated exchange. $T_{1}$ is shorter ( $\left.150 \mathrm{~ms}\right)$ than in the slightly decomposed sample $(\sim 400 \mathrm{~ms})$ most probably because of the lower amount of free water; the free water component in the $T_{2}$ distributions appears to be much smaller for piece 1 than for piece 20. However, the changes in the micropore composition may also have their own contribution to the relaxation times.

$T_{1}$ values of the slightly decomposed, water-saturated sample were much longer than in the other slightly decomposed sample due to much higher amount of free water. $T_{2}$ values of the free water component were also slightly longer for the same reason. Note that the large signal on the left edge of the $T_{2}$ distribution is most probably an artifact related to the instabilities of Laplace inversion; such artefacts are typical on edges (Bernin and Topgaard 2013).

In addition to the above-mentioned effects, sugars and other cell wall degradation products in the liquid phase will affect the $T_{2}$ and $T_{1}$ of the liquid (Labbe et al. 2006).

In summary, the decomposition of wood is reflected in the relaxation distributions in the following way: $T_{2}$ relaxation time of bound water is increased due to increased mobility; $T_{1}$ relaxation times of free and bound water components become equal as the exchange between these pools is accelerated due to the destruction of the structure of wood; when the sample is fully decomposed, $T_{1}$ becomes shorter because of the lowered amount of free water and changed pore structure.

\section{Full time series}

Samples 1 to 3 were analyzed by MRI and gravimetrically altogether six times during the decay period. The full time series of the analysis is shown in Figure 5. All those samples belong to the class "decomposed".

During the first week, the mass of the samples doubled due to the adsorption of the moisture from the humid atmosphere of the incubation cabinet, the moisture brought by the fungus and produced in the decomposition process as well as by the growth of hypha (as explained in Materials and methods, the initial MC of the samples was $\sim 2 \%)$. In MRI taken in the first week, intense free water signals were visible only on the surfaces of the samples. In the second week, intense free water signals were visible in the inner part of the sample, showing the progress of the decay process. Between weeks 2 and 4, the masses of the samples decreased substantially, and MRI contained mostly noise. This implies that the samples dried, and the fungus could not work efficiently due to lack of water. Thereafter, the dishes were sealed better with parafilm (see Materials and methods) to prevent 

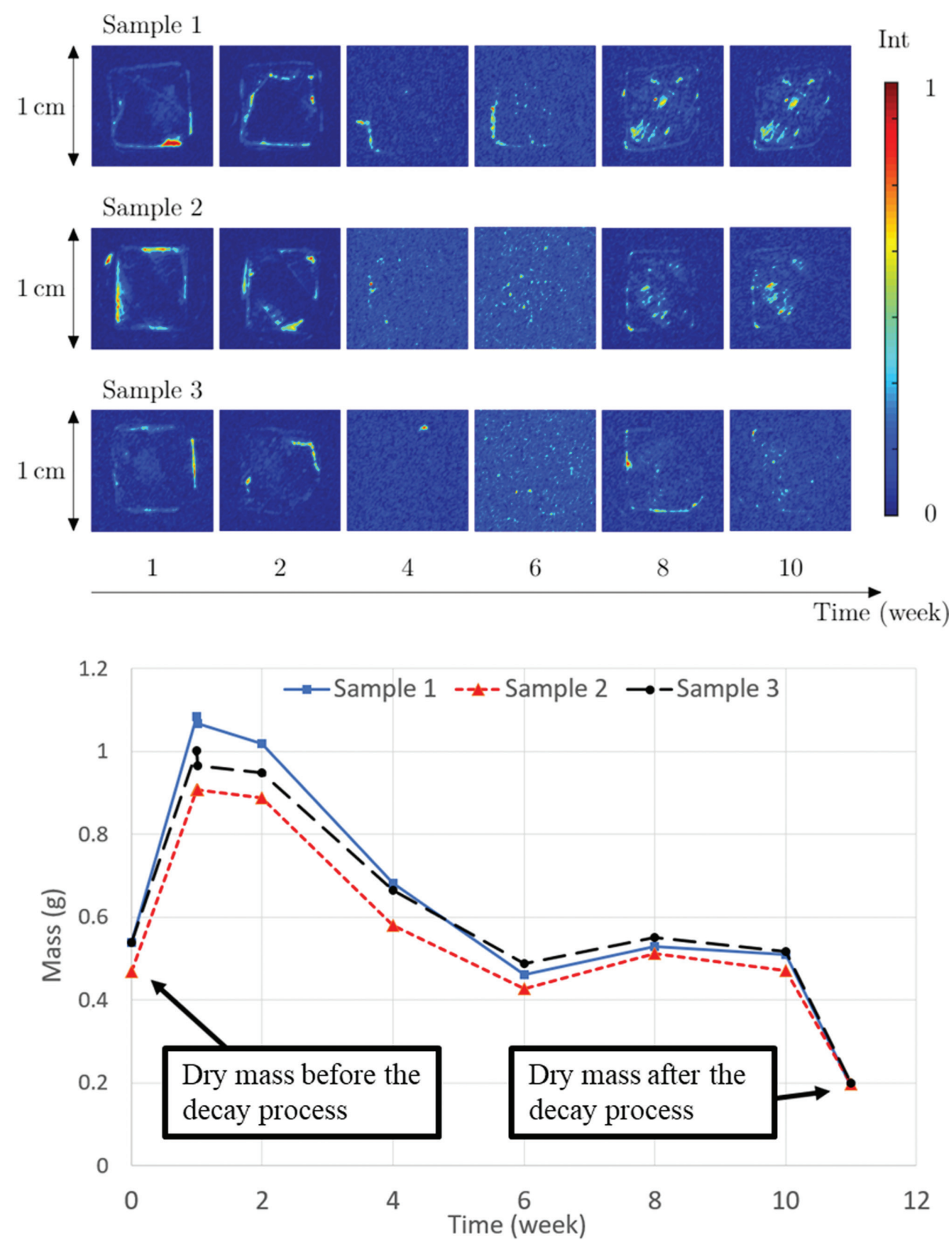

Figure 5: Spin-density MRI (top) and mass (bottom) of wood samples 1 to 3 as a function of decay time.

Note that the first and last points correspond to the dry masses of the samples before and after the decay processes, whereas the other masses are the wet masses.

samples from drying. As a result, the weight of the sample became slightly higher on weeks 8 and 10, and free water was observed again in MRI. The intense free water spots show that the fungus was active deep inside the samples. The final dry mass after the decay process was about one third of the initial dry mass, reflecting the significant decomposition of the cellulose and hemicellulose of the sample.

In summary, MRI clearly showed the initial active regions of the fungus, although it could not be observed from the gravimetric data yet. The images also visualize how the decay process progressed deeper inside the samples, and the lack of free water signal revealed the period of the low activity of the fungus due to too low MC. In contrast, the images showed also that the activity of the fungus was recovered after raising the MC of the sample.

\section{Conclusions}

This article demonstrated the usefulness of the combined MRI and relaxation analysis for monitoring the decay 
process of wood in detail. MRI reveals the active regions of the fungus through the observation of free water brought by the fungus and produced in the decomposition process. MRI shows the initial activity of the fungus and visualizes the progress of the decomposition process toward the interior of the sample. $T_{1}-T_{2}$ correlation measurements highlight the changes in the mobility and exchange of water molecules due to the decomposition, providing important information on the microstructure of wood. The decomposition of the cell walls is reflected as elongated $T_{2}$ value of bound water, and it also made the $T_{1}$ values of all the components equal due to accelerated exchange between the water pools. Overall, the results, interpretations, and classifications reported in this article facilitate the use of these methods in the characterization of the decomposition process noninvasively in real time. The methods might be used, for example, in the investigation of new kinds of protective agents of wood or in situ detection of decomposition of buildings by portable NMR/MRI devices (Dvinskikh et al. 2011a,b; Pourmand et al. 2011).

Acknowledgements: The authors are grateful to the Research Unit of Ecology and Genetics at the University of Oulu (Saija Ahonen and Annamari Markkola) for the access to the incubation cabinet and help. We are also grateful to the Laplace inversion program provided by the late Prof. P. Callaghan.

Author contributions: All the authors have accepted responsibility for the entire content of this submitted manuscript and approved submission.

Research funding: We acknowledge the financial support of the European Research Council (ERC) under Horizon 2020 (H2020/2018-2022/ERC grant agreement no. 772110, Funder Id: http://dx.doi.org/10.13039/100010663), Academy of Finland (grants. 289649, 294027, and 319216, Funder Id: http://dx.doi.org/10.13039/501100002341), and the Kvantum Institute (University of Oulu).

Employment or leadership: None declared.

Honorarium: None declared.

\section{References}

Almeida, G., Gagné, S., Hernández, E. (2007) A NMR study of water distribution in hardwoods at several equilibrium moisture contents. Wood Sci. Technol. 41:293-307.

Araujo, C.D., Mackay, A.L., Hailey, J.R.T., Whittall, K.P., Le, H. (1992) Proton magnetic resonance techniques for characterization of water in wood: application to white Spruce. Wood Sci. Technol. 26:101-113.
Beck, G., Thybring, E.E., Thygesen, L.G., Hill, C. (2017) Characterization of moisture in acetylated and propionylated radiata pine using low-field nuclear magnetic resonance (LFNMR) relaxometry. Holzforschung 72:225-233.

Beck, G., Thybring, E.E., Thygesen, L.G. (2018) Brown-rot fungal degradation and de-acetylation of acetylated wood. Int. Biodeter. Biodegr. 135:62-70.

Bernin, D., Topgaard, D. (2013) NMR diffusion and relaxation correlation methods: new insights in heterogeneous materials. Curr. Opin. Colloid Interface Sci. 18:166-172.

Bonnet, M., Courtier-Murias, D., Faure, P., Rodts, S., Care, S. (2017) NMR determination of sorption isotherms in earlywood and latewood of Douglas fir. Identification of bound water components related to their local environment. Holzforschung 71:481-490.

Brown, R.W., Cheng, Y.-U.N., Haacke, E.M., Thompson, M.R., Venkatesan, R. Magnetic Resonance Imaging: Physical Principles and Sequence Design. Wiley, New Jersey, 2016.

Brownstein, K.R., Tarr, C.E. (1979) Importance of classical diffusion in NMR studies of water in biological cells. Phys. Rev. A 19:2446-2453.

Carr, H.Y., Purcell, E.M. (1954) Effects of diffusion on free precession in nuclear magnetic resonance experiments. Phys. Rev. 93:630-638.

Cox, J., McDonald, P.J., Gardiner, B.A. (2010) A study of water exchange in wood by means of 2D NMR relaxation correlation and exchange. Holzforschung 64:259-266.

Dvinskikh, S.V., Henriksson, M., Berglund, L.A., Furó, I. (2011a) A multinuclear magnetic resonance imaging (MRI) study of wood with absorbed water: estimating bound water concentration and local wood density. Holzforschung 65:103-107.

Dvinskikh, S.V., Furó, I., Sandberg, D., Söderström, O. (2011b) Moisture content profiles and uptake kinetics in wood cladding materials evaluated by a portable nuclear magnetic resonance spectrometer. Wood Mat. Sci. Eng. 6:119-127.

European standard EN 113 (1996) Wood preservatives - test method for determining the protective effectiveness against wood destroying basidiomycetes - Determination of the toxic values. European Committee for Standardization, Brussels. p. 31.

Fantazzini, P., Maccotta, A., Gombia, M., Garavaglia, C., Brown, R.J.S., Brai, M. (2006) Solid-liquid nuclear magnetic resonance relaxation and signal amplitude relationships with ranking of seasoned softwoods and hardwoods. J. Appl. Phys. 100:074907.

Fredriksson, M., Thygesen, L.G. (2017) The states of water in Norway spruce (Picea abies (L.) Karst.) studied by low-field nuclear magnetic resonance (LFNMR) relaxometry: assignment of free-water populations based on quantitative wood anatomy. Holzforschung 71:77-90.

Gilardi, G., Abis, L., Cass, A.E. (1994) Wide-line solid-state NMR of wood: proton relaxation time measurements on cell walls biodegraded by white-rot and brown-rot fungi. Enzyme Microb. Technol. 16:676-682.

Gilardi, G., Abis, L., Cass, A.E. (1995) Carbon-13 CP/MAS solid-state NMR and FT-IR spectroscopy of wood cell wall biodegradation. Enzyme Microb. Technol. 17:268-275.

Griffin, D. (1977) Water potential and wood-decay fungi. Annu. Rev. Phytopathol. 15:319-329.

Hameury, S., Sterley, M. (2006) Magnetic resonance imaging of moisture distribution in Pinus sylvestris L. exposed to daily 
indoor relative humidity fluctuations. Wood Mater. Sci. Eng. 1:116-126.

Hartley, I.D., Kamke, F.A., Peemoeller, H. (1994) Absolute moisture content determination of aspen wood below the fiber saturation point using pulsed NMR. Holzforschung 48:474-479.

Hernández, R.E., Cáceres, C.B. (2010) Magnetic resonance microimaging of liquid water distribution in sugar maple wood below fiber saturation point. Wood Fiber Sci. 42:259-272.

Hoffmeyer, P., Engelund, E.T., Thygesen, L.G. (2011) Equilibrium moisture content (EMC) in Norway spruce during the first and second desorptions. Holzforschung 65:875-882.

Hyde, S.M., Wood, P.M. (1997) A mechanism for production of hydroxyl radicals by the brown-rot fungus Coniophora puteana: Fe(III) reduction by cellobiose dehydrogenase and $\mathrm{Fe}(\mathrm{II})$ oxidation at a distance from the hyphae. Microbiology 143:259-266.

Ibach, R., Sun, G., Gnatowski, M., Glaeser, J., Leung, M., Haight, J. (2016) Exterior decay of wood-plastic composite boards: characterization and magnetic resonance imaging. Forest Prod. J. 66:4-17.

Irbe, I., Andersons, B., Chirkova, J., Kallavus, U., Andersone, I., Faix, O. (2006) On the changes of pinewood (Pinus sylvestris L.) chemical composition and ultrastructure during the attack by brown-rot fungi Postia placenta and Coniophora puteana. Int. Biodeter. Biodegr. 57:99-106.

Javed, M.A., Kekkonen, P.M., Ahola, S., Telkki, V.-V. (2015) Magnetic resonance imaging study of water absorption in thermally modified pine wood. Holzforschung 69:899-907.

Karppanen, O., Venäläinen, M., Harju, A.M., Laakso, T. (2008) The effect of brown-rot decay on water adsorption and chemical composition of Scots pine heartwood. Ann. For. Sci. 65:610.

Keeler, J. Understanding NMR Spectroscopy. Chichester, Wiley, 2010.

Kekkonen, P.M., Telkki, V.-V., Jokisaari, J. (2009) Determining the highly anisotropic cell structures of Pinus sylvestris in three orthogonal directions by PGSTE NMR of absorbed free water and methane. J. Phys. Chem. B 113:1080-1084.

Kekkonen, P.M., Ylisassi, A., Telkki, V.-V. (2014) Absorption of water in thermally modified pine wood as studied by NMR. J. Phys. Chem. C 118:2146-2153.

Kettunen, P.O. (2006) Structure and Properties of Wood. Trans Tech Publications Ltd., Durnten-Zurich, Switzerland.

Koenig, A.B., Sleighter, R.L., Salmon, E., Hatcher, P.G. (2010) NMR structural characterization of Quercus alba (white oak) degraded by the brown rot fungus, Laetiporus sulphureus. J. Wood Chem. Technol. 30:61-85.

Labbé, N., De Jéso, B., Lartigue, J.-C., Daudé, G., Pétraud, M., Ratier, M. (2002) Moisture content and extractive materials in maritime pine wood by low field ${ }^{1} \mathrm{H}$ NMR. Holzforschung 56:25-31.

Labbé, N., De Jéso, B., Lartigue, J., Daudé, G., Pétraud, M., Ratier, M. (2006) Time-domain $1 \mathrm{H}$ NMR characterization of the liquid phase in greenwood. Holzforschung 60:265-270.

MacMillan, M.B., Schneider, M.H., Sharp, A.R., Balcom, B.J. (2002) Magnetic resonance imaging of water concentration in low moisture content wood. Wood Fiber Sci. 34:276-286.

Mazela, B., Kowalczuk, J., Ratajczak, I., Szentner, K. (2014) Moisture content (MC) and multinuclear magnetic resonance imaging (MRI) study of water absorption effect on wood treated with aminofunctional silane. Eur. J. Wood Prod. 72:243-248.
Meiboom, S., Gill, D. (1958) Modified spin-echo method for measuring nuclear relaxation times. Review of scientific instruments. 29:688-691.

Menon, R.S., MacKay, A.L., Hailey, J.R.T., Bloom, M., Burgess, A.E., Swanson, J.S. (1987) An NMR determination of the physiological water distribution in wood during drying. J. Appl. Polymer Sci. 33:1141-1155.

Menon, R.S., MacKay, A.L., Filbotte, S., Hailey, J.R. (1989) Quantitative separation of NMR images of water in wood on the basis of $T_{2}$. J. Magn. Reson. 82:205-210.

Merela, M., Oven, P., Serša, I., Mikac, U. (2009) A single point NMR method for an instantaneous determination of the moisture content of wood. Holzforschung 63:348-351.

Müller, U., Bammer, R., Halmschlager, E., Stollberger, R., Wimmer, R. (2001) Detection of fungal wood decay using magnetic resonance imaging. Eur. J. Wood Wood Prod. 59:190-194.

Novaes, E., Kirst, M., Chiang, V., Winter-Sederoff, H., Sederoff, R. (2010) Lignin and biomass: a negative correlation for wood formation and lignin content in trees. Plant Physiol. 154:555-561.

Onuchin, E., Medyakov, A., Grunin, L., Sidorova, E. (2018) A study of the structure of wood damaged by rot using nuclear magnetic resonance. J. Appl. Eng. Sci. 16:258-262.

Pearce, R.B., Fisher, B.J., Carpenter, T.A., Hall, L.D. (1997) Water distribution in fungal lesions in the wood of sycamore, Acer pseudoplatanus, determined gravimetrically and using nuclear magnetic resonance imaging. New Phytol. 135:675-688.

Peemoeller, H., Shenoy, R.K., Pintar, M.M. (1981) Two-dimensional NMR time evolution correlation spectroscopy in wet lysozyme. J. Magn. Reson. 45:193-204.

Pourmand, P., Wang, L., Dvinskikh, S.V. (2011) Assessment of moisture protective properties of wood coatings by a portable NMR sensor. J. Coat. Technol. Res. 8:649-654.

Quick, J.J., Hailey, J.R.T., Mackay, A.L. (1990) Radial moisture profiles of cedar sapwood during drying a proton magnetic resonance study. Wood Fiber Sci. 22:404-412.

Riggin, M.T., Sharp, A.R., Kaiser, R. (1979) Transverse NMR relaxation of water in wood. J. Appl. Polym. Sci. 23: 3147-3154.

Ringman, R., Beck, G., Pilgård, A. (2019) The importance of moisture for brown rot degradation of modified wood: a critical discussion. Forests 10:522.

Rosenkilde, A., Glover, P. (2002) High resolution measurement of the surface layer moisture content during drying of wood using a novel magnetic resonance imaging technique. Holzforschung 56:312-317.

Rostom, L., Courtier-Murias, D., Rodts, S., Care, S. (2019) Investigation of the effect of aging on wood hygroscopicity by $2 \mathrm{D}^{1} \mathrm{H}$ NMR relaxometry. Holzforschung, DOI: https://doi.org/10.1515/ hf-2019-0052.

Sharp, A.R., Riggin, M.T., Kaiser, R., Schneider, M.H. (1978) Determination of moisture content of wood by pulsed nuclear magnetic resonance. Wood Fiber Sci. 10:74-81.

Siau, J.S. Transport Processes in Wood. Springer-Verlag, Berlin, 1984. Simpson, W. (2007) Sorption theories applied to wood. Wood Fiber Sci. 12:183-195.

Sivonen, H., Maunu, S.L., Sundholm, F., Jämsä, S., Viitaniemi, P. (2002) Magnetic resonance studies of thermally modified wood. Holzforschung 56:648-654. 
Skaar, C. Wood-Water Relations. Springer-Verlag, Berlin, 1988.

Song, Y.-Q., Venkataramanan, L., Hürlimann, M.D., Flaum, M., Frulla, P., Straley, C. (2002) T1-T2 correlation spectra obtained using a fast two-dimensional Laplace inversion. J. Magn. Reson. 154:261-268.

Telkki, V.-V., Yliniemi, M., Jokisaari, J. (2013) Moisture in softwoods: fiber saturation point, hydroxyl site content, and the amount of micropores as determined from NMR relaxation time distributions. Holzforschung 67:291-300.

Terenzi, C., Dvinskikh, S.V., Furo, I. (2013) Wood microstructure explored by anisotropic $1 \mathrm{H}$ NMR line broadening: experiments and numerical simulations. J. Phys. Chem. B 117:8620-8632.

Thygesen, L.G., Elder, T. (2009) Moisture in untreated, acetylated, and furfurylated Norway spruce studied during drying using time domain NMR. Wood Fiber Sci. 41:194-200.

Tura, D., Wasser, S.P., Zmitrovich, I.V. (2016) Wood-inhabiting fungi: applied aspects. Fungi: Applications and Management Strategies. 245.

van Houts, J.H., Wang, S., Shi, H., Pan, H., Kabalka, G.W. (2004) Moisture movement and thickness swelling in oriented strandboard, Part 1. Analysis using nuclear magnetic resonance microimaging. Wood Sci. Technol. 38:617-628.

van Houts, J.H., Wang, S., Shi, H., Kabalka, G.W. (2006) Moisture movement and thickness swelling in oriented strandboard.
Part 2: analysis using nuclear magnetic resonance imaging body scanner. Wood Sci. Technol. 40:437-443.

Vold, R.L., Waugh, J.S., Klein, M.P., Phelps, D.E. (1968) Measurement of spin relaxation in complex systems. J. Chem. Phys. 48:3831-3832.

Wang, P.C., Chang, S.J. (2007) Nuclear magnetic resonance imaging of wood. Wood Fiber Sci. 18:308-314.

Williams, F.C., Hale, M. (2003) The resistance of wood chemically modified with isocyanates: the role of moisture content in decay suppression. Int. Biodeter. Biodegr. 52: 215-221.

Zabel, R.A., Morrell, J.J. Wood Microbiology: Decay and its Prevention. Academic Press, San Diego, CA, USA, 1992.

Zhang, M., Wang, X., Gazo, R. (2013) Water states in yellow poplar during drying studied by time-domain nuclear magnetic resonance. Wood Fiber Sci. 45:423-428.

Zhou, M., Caré, S., Courtier-Murias, D., Faure, P., Rodts, S., Coussot, P. (2018) Magnetic resonance imaging evidences of the impact of water sorption on hardwood capillary imbibition dynamics. Wood Sci. Technol. 52:929-955.

Zupanc, M.Z., Mikac, U., Sersa, I., Merela, M., Humar, M. (2019) Water distribution in wood after short term wetting. Cellulose 26:703-721. 